\title{
Controlling Mechanism of Molecular Orientation of Poly(3- alkylthiophene) in a Thin Film Revealed by Using pMAIRS
}

\section{$\operatorname{AUTHOR}(S)$ :}

Shioya, Nobutaka; Shimoaka, Takafumi; Eda, Kazuo; Hasegawa, Takeshi

\section{CITATION:}

Shioya, Nobutaka ... [et al]. Controlling Mechanism of Molecular Orientation of Poly(3alkylthiophene) in a Thin Film Revealed by Using pMAIRS. Macromolecules 2017, 50(13): 5090-5097

\section{ISSUE DATE:}

2017-06-27

URL:

http://hdl.handle.net/2433/226493

\section{RIGHT:}

This document is the Accepted Manuscript version of a Published Work that appeared in final form in 'Macromolecules', copyright (c) American Chemical Society after peer review and technical editing by the publisher. To access the final edited and published work see http://doi.org/10.1021/acs.macromol.7b00826:; The full-text file will be made open to the public on 27 June 2018 in accordance with publisher's 'Terms and Conditions for Self-Archiving'.; この論文は出版社 版でありません。引用の際には出版社版をご確認ご利用ください。; This is not the published version. Please cite only the published version. 


\section{Controlling mechanism of molecular orientation of poly(3-alkylthiophene) in a thin film revealed by using PMAIRS}

Nobutaka Shioya, ${ }^{\dagger}$ Takafumi Shimoaka, ${ }^{\dagger}$ Kazuo Eda, ${ }^{\ddagger}$ and Takeshi Hasegawa ${ }^{* \dagger}$

${ }^{\dagger}$ Laboratory of Solution and Interface Chemistry, Division of Environmental Chemistry, Institute for Chemical Research, Kyoto University, Gokasho, Uji, Kyoto 611-0011, Japan

tDepatment of Chemistry, Graduate School of Science, Kobe University, 1-1 Rokko-dai, Nada-ku, Kobe, Hyogo 657-8501, Japan

\footnotetext{
${ }^{\dagger}$ To whom correspondence should be addressed.

E-mail: htakeshi@scl.kyoto-u.ac.jp
} 


\section{$\underline{\text { TOC (for Table of Contents use only) }}$}

"Controlling mechanism of molecular orientation of poly(3-alkylthiophene) in a thin film revealed by using pMAIRS"

by Nobutaka Shioya, Takafumi Shimoaka, Kazuo Eda, and Takeshi Hasegawa

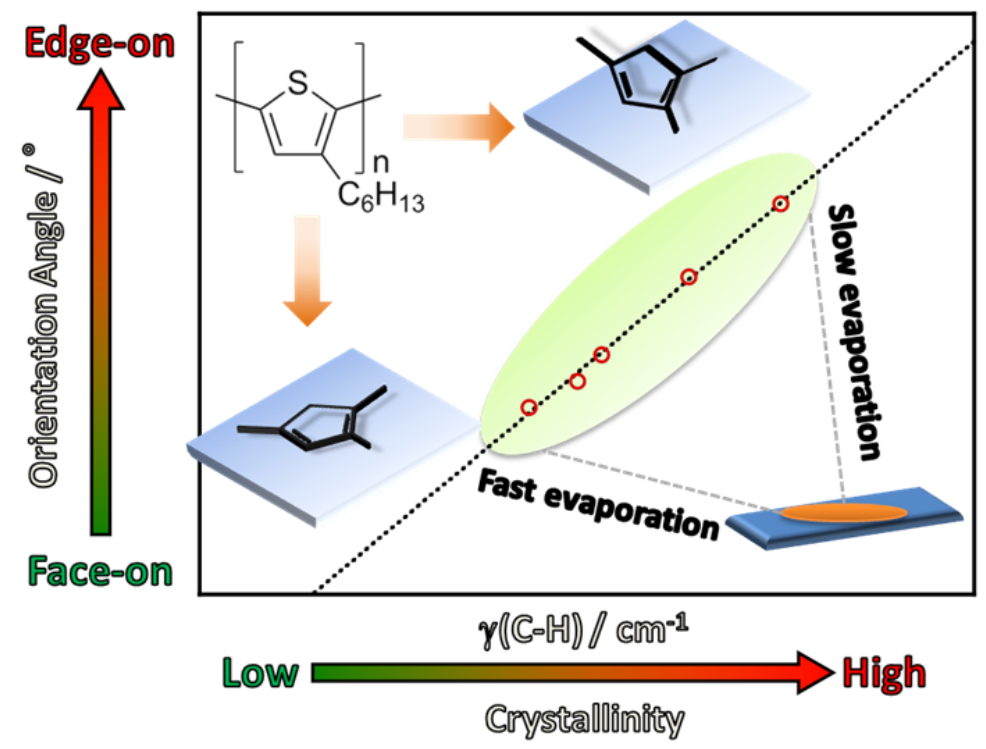


ABSTRACT: A face-on oriented thin film of poly(3-hexylthiophene) (P3HT) is suitable for an organic semiconductor layer in a photovoltaic device, and thus analysis of the film structure in terms of molecular orientation is crucial. Although the face-on film often has a poor crystallinity, diffraction techniques have long been employed for the structural analysis, and only very minor crystal parts have been discussed. In our previous study, P3HT was revealed to have a uniquely oriented structure even in an amorphous film by using p-polarized multiple-angle incidence resolution spectrometry (pMAIRS), which is powerful to analyze the molecular orientation, crystallinity, and the conjugation length of P3HT. With the best use of the potential of pMAIRS, in the present study, the controlling mechanism of the molecular orientation of P3HT correlated with the crystallinity in a spin-coated thin film is revealed. As a result, by employing a high-spin speed at $8000 \mathrm{rpm}$, a highly face-on oriented thin film having a very low crystallinity is obtained, which readily reveals that the face-on component has a strong correlation with the amorphous aggregates. 


\section{INTRODUCTION}

Polythiophene is a $\pi$-conjugated polymer, which absorbs visible light, and therefore its derivatives are employed for an active layer in organic photoelectric devices. Since poly(3-hexylthiophene) (P3HT) having a hexyl chain on each thiophene ring exhibits the highest device performance as a p-type semiconductor among poly(3-alkylthiophene)s (P3ATs), ${ }^{1-4}$ a thin film of P3HT is often incorporated into organic field effect transistors ${ }^{5}$ and organic photovoltaic devices. ${ }^{6}$ By attaching an alkyl chain to the 3-position of the thiophene ring, the thiophene ring takes a coplanar structure in the thin film due to the zippering effect between the adjacent alkyl chains, ${ }^{7,8}$ which makes the efficient of electron transfer higher.

$\mathrm{P} 3 \mathrm{HT}$ is also known as a semi-crystalline polymer that is composed of crystal and amorphous components. ${ }^{9-11}$ In former studies, ${ }^{12,13}$ the ring-parallel (face-on) and the ring-vertical (edge-on) orientations are found to be generated in the crystal region of the thin film, in which the molecules are in a lamellar structure. In the amorphous region, on the other hand, the molecules have long been believed to be nearly random. This schematic image of the amorphous region was, however, not on an experimental fact, since an X-ray diffraction (XRD) analysis that needs a crystal has long been used as the first choice to discuss the film structure. 
This analytical limit can be overcome by employing p-polarized multiple-angle incidence resolution spectrometry (pMAIRS), ${ }^{14-17}$ which is a powerful technique to discuss the molecular structure even in an amorphous region. ${ }^{18,19} \mathrm{P} 3 \mathrm{HT}$ works as a semiconductor even with a low crystallinity in the film, and the aptitude for an electronic device strongly depends on the molecular orientation and its distribution. ${ }^{12,20-23}$ In organic photovoltaic devices, for example, the face-on oriented thin film is favored, although it exhibits a low crystallinity. Infrared (IR) pMAIRS is therefore promising for discussing the molecular orientation in a P3HT thin film particularly with a low crystallinity. In fact, we have recently revealed ${ }^{8}$ that P3HT has a unique molecular orientation structure in an amorphous film using the pMAIRS technique: a short-axis of the polythiophene backbone is kept highly oriented parallel to the substrate surface; whereas the long-axis is nearly disordered, which is totally different from the conventional image based on XRD analyses.

A key factor to control the molecular arrangement involving the orientation in a casted film has recently been found to have a strong correlation with the evaporation 'time' of a solvent that depends on the volatile character of solvent and the preparation technique, since the evaporation time directly influences the crystallinity. ${ }^{24,25}$ The edge-on orientation of $\mathrm{P} 3 \mathrm{HT}$, for instance, is induced when a slowly evaporating solvent 
represented by 1,2,4-trichlorobenzene (TCB) is used. ${ }^{26,27}$ In contrast, the face-on oriented film is prepared by using a fast evaporating solvent like chloroform (Chl) with a high-spin speed $(>2000 \mathrm{rpm}){ }^{28}$ The correlation between the face-on orientation and the crystallinity is, however, not fully understood, since the face-on aggregate is generally regarded as the 'crystallites,' which is inconsistent with a fact that the face-on oriented thin film often exhibits low crystallinity.

In the present study, the structure of a P3HT film is controlled by systematically changing the combination of the solvent and the spin speed, which influences the crystallinity in the film. Then, the correlation between the molecular orientation and the crystallinity is discussed on pMAIRS spectra. As a result, a very simple and useful linear correlation has been revealed: the face-on component increases in the thin film as the crystallinity decreases. This apparently indicates that the major component of the face-on orientation is correlated with the 'amorphous' portion; whereas the edge-on orientation is driven by the crystallization of P3HT. In addition, poly(3-butylthiophene) (P3BT) having a shorter alkyl chain having a lower degree of crystallinity is also studied, which confirms that the face-on stance is generated in an amorphous region. To achieve the face-on oriented thin film with high crystallinity, therefore, mechanical techniques using shear forces such as rubbing and friction-transfer methods are 
employed. $^{29,30}$

\section{EXPERIMENTAL METHODS}

Sample preparation: Regioregular (regioregularity $>95 \%, M_{\mathrm{n}}: 15,000-45,000$ ) P3HT was purchased from Aldrich (Milwaukee, WI, USA). Regioregular P3BT was obtained from Alfa Aesar (Ward Hill, MA, USA). They were used without further purification. Chloroform (Chl) of ACS Spectra Grade ( $\geq 99.8 \%)$ and 1,2,4-trichlorobenzene (TCB) with a purity of $\geq 99 \%$ were purchased from Sigma-Aldrich (St. Louis, MO, USA). The spin-coated (SC) films were prepared on a Si substrate from the Chl and TCB solutions with a concentration within a range of $5.0-10.0 \mathrm{mg} \mathrm{mL}^{-1}$, which are denoted as Chl-SC and TCB-SC films, respectively. The TCB-SC film was spin-coated at $1500 \mathrm{rpm}$. The spin coating of the Chl-SC film was carried out at various spin-speeds of 1000, 2000, 4000 and $8000 \mathrm{rpm}$. Since P3BT has a poor solubility, ${ }^{8}$ on the other hand, the Chl solution was heated at ca. $70^{\circ} \mathrm{C}$ for $20 \mathrm{~min}$ for complete dissolution, and then the spin-coated film was prepared with the warm solution. The Si wafer with a thickness of $0.675 \pm 0.025 \mathrm{~mm}$ was provided by Valqua FFT (Tokyo, Japan). To confirm the reproducibility of the spin-coated films, the film preparation and pMAIRS measurements were both repeated three times for each spin-speed.

IR pMAIRS measurements: IR pMAIRS measurements were carried out on a 
Thermo Fischer Scientific (Madison, WI, USA) Nicolet 6700 FT-IR spectrometer equipped with a Thermo Fischer Scientific (Yokohama, Japan) automatic MAIRS equipment (TN10-1500). The incident light was polarized to have the p-polarization via a Harrick (Pleasantville, NY, USA) PWG-U1R wire-grid polarizer, and the transmitted light through the sample was detected by a MCT detector. The wavenumber resolution was $4 \mathrm{~cm}^{-1}$. The optimal angles of incidence that are from $9^{\circ}$ to $44^{\circ}$ by $5^{\circ}$ steps ${ }^{16}$ were employed, and the interferogram was accumulated 1000 times for each angle of incidence.

pMAIRS spectra provide the orientation angle, $\phi$, of a transition moment averaged on a wide area of the thin film of ca. $2 \mathrm{~cm}^{2}$, which is simply calculated by using the dichroic ratio of IP (in-plane) to OP (out-of-plane) spectra $\left(A_{\mathrm{IP}} / A_{\mathrm{OP}}\right)$ as follows: ${ }^{31}$

$$
\phi=\tan ^{-1} \sqrt{\frac{2 A_{\mathrm{IP}}}{A_{\mathrm{OP}}}},
$$

where $\phi$ is the angle from the surface normal. In the present study, the analytical error of $\phi$ due to the spectral noise is less than $\pm 1^{\circ} .^{24}$ 
UV-vis measurements: UV-vis spectra were measured on a Jasco (Tokyo, Japan) V-630 UV-vis spectrometer. The samples were Chl-SC and TCB-SC films prepared on a $\mathrm{CaF}_{2}$ substrate with a size of $40 \times 20 \mathrm{~mm}^{2}$. The substrate was purchased from Pier Optics (Gunma, Japan).

GIXD measurements: Grazing incidence X-ray diffraction (GIXD) analysis was performed on a RIGAKU (Tokyo, Japan) SuperLab diffractometer with a $\mathrm{Cu}$ rotating anode X-ray generator. The incident light was $\mathrm{Cu}-\mathrm{K} \alpha$ radiation $(\lambda=0.15418 \mathrm{~nm})$ generated at $40 \mathrm{kV}$ and $30 \mathrm{~mA}$. The angle between the incidental $\mathrm{X}$-ray and the substrate was fixed at $0.20^{\circ}$. The diffraction patterns with the in-plane (IP) and out-of-plane (OP) geometries were measured with a scan speed of $1^{\circ} \min ^{-1}$ by $0.01^{\circ}$ steps.

\section{RESULTS AND DISCUSSION}

Molecular orientation of P3HT in a spin-coated thin film: Figure 1 presents pMAIRS spectra of P3HT thin films prepared as a function of the solvent evaporation time, which can be controlled by changing the combination of the solvent and the film preparation method. Since both $\mathrm{Chl}$ and SC have a fast solvent-evaporation character, 
for example, the combination of Chl-SC gives a very short evaporation time. ${ }^{24}$ In this paper, the films are systematically named as Chl-SC- $X$ ( $X$; spin-speed). Since the TCB needs a long evaporation time to have a dried film due to the low-volatile character, $^{24,26,32}$ the spin speed is fixed at $1500 \mathrm{rpm}$ for the TCB-SC series. Since the evaporation time of the Chl-SC film, on the other hand, is a relatively short, the time can be controlled by changing the spin speed, $X$. For example, the Chl-SC-8000 film has the shortest evaporation time in our P3HT films (Figure 1e).

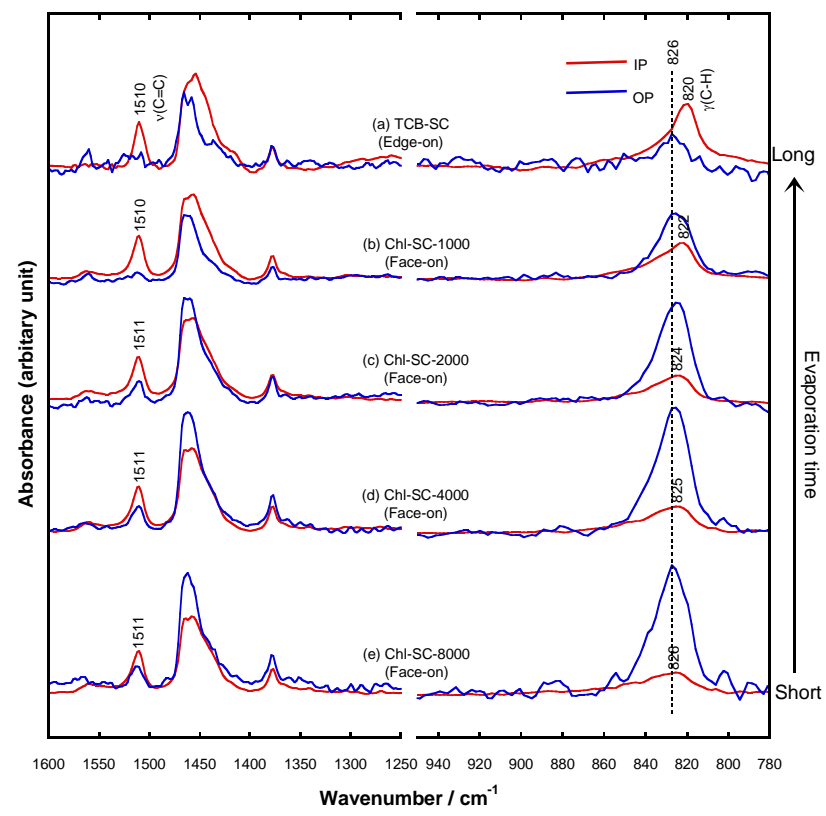

Figure 1 Representative pMAIRS spectra of P3HT thin films prepared as a function of solvent and spin-speed: (a) TCB-SC, (b) Chl-SC-1000, (c) Chl-SC-2000, (d) Chl-SC-4000, and (e) Chl-SC-8000 films. The red and blue lines correspond to IP and OP spectra, respectively.

In our previous studies, ${ }^{8,27}$ two bands at ca. $1510 \mathrm{~cm}^{-1}$ and ca. $820 \mathrm{~cm}^{-1}$, which are assigned to the ring anti-symmetric stretching vibration $(v(\mathrm{C}=\mathrm{C}))$ and the $\mathrm{C}-\mathrm{H}$ 
out-of-plane deformation vibration $(\gamma(\mathrm{C}-\mathrm{H}))$ modes of the thiophene ring, respectively, are found to be orientation-sensitive marker bands of P3HT. The transition moment of the $v(C=C)$ mode is parallel to the polymer chain; while the $\gamma(\mathrm{C}-\mathrm{H})$ mode has a perpendicular transition moment to the thiophene ring. In the case of the face-on orientation, therefore, the $v(\mathrm{C}=\mathrm{C})$ band in the IP spectrum is larger than that in the OP spectrum; while the $\gamma(\mathrm{C}-\mathrm{H})$ band appears dominantly in the OP spectrum. The edge-on oriented film, on the other hand, exhibits larger IP bands for both $v(\mathrm{C}=\mathrm{C})$ and $\gamma(\mathrm{C}-\mathrm{H})$ modes than OP bands.

Considering this surface-selection rule, the TCB-SC film (Figure 1a) is categorized into the edge-on orientation; whereas the Chl-SC films (Figure 1b-e) are found to have the face-on orientation. ${ }^{8,27}$ In addition, the band intensity ratio of the $\gamma(\mathrm{C}-$ H) mode in the IP spectrum to that in the OP spectrum becomes larger as the spin speed is made lower. In this manner, the molecular orientation looks correlated with the evaporation time through the control of the combination of solvent and spin speed. The solvent evaporation time is thus a key parameter for controlling the molecular orientation of $\mathrm{P} 3 \mathrm{HT}^{28}$ This trend will readily be quantitatively confirmed as follows.

To quantitatively discuss the IR results mentioned above, the orientation angle of the thiophene ring is calculated. Details of the calculation are referred to literature. ${ }^{8}$ 
Figure 2 shows the orientation angle of the $\gamma(\mathrm{C}-\mathrm{H})$ mode from the surface normal, $\phi_{\mathrm{C}-\mathrm{H}}$, as a function of the spin speed. This angle can be interpreted as the tilt angle of the thiophene ring to the substrate surface. ${ }^{8}$ Therefore, if the P3HT molecule takes the ideal face-on orientation in the film, $\phi_{\mathrm{C}-\mathrm{H}}=0^{\circ}$ would be yielded; while $\phi_{\mathrm{C}-\mathrm{H}}=90^{\circ}$ would be yielded for the ideal edge-on orientation.

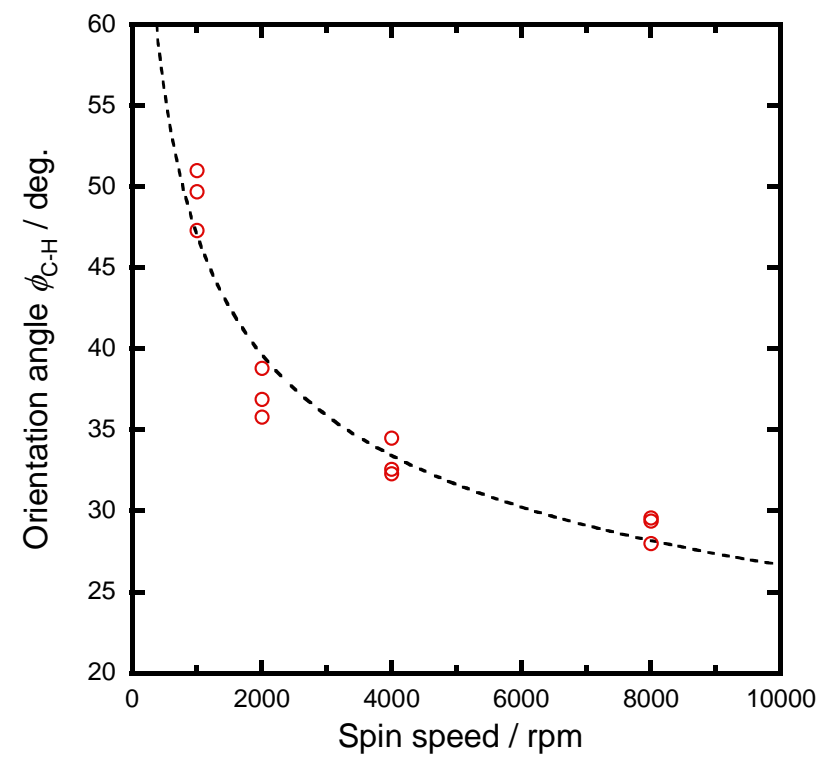

Figure 2 Plot of the orientation angle, $\phi_{\mathrm{C}-\mathrm{H}}$, against spin-speed. Three films are measured for each spin-speed to check the reproducibility.

As presented in Figure 2, the decay curve of the correlation of $\phi_{\mathrm{C}-\mathrm{H}}$ with the spin speed indicates a monotonous decrease as the spin speed becomes higher. The three points at each spin speed are of the repeated measurements for checking the reproducibility. The decreasing trend can be interpreted that the face-on orientation component is increased when the solvent evaporation time is made shorter; while the 
slow-evaporation enhances the edge-on orientation through the crystallite generation, which is in good agreement with former studies..$^{10,28}$

It should be mentioned that the SC-1000 film gives the angle of about $50^{\circ}$ that is closed to a magic angle $\left(54.7^{\circ}\right)$ that corresponds to an isotropic structure. However, the molecules in the film are not randomly orientated, but they have $\phi_{\mathrm{C}-\mathrm{H}} \approx 50^{\circ}$. If the film is isotropic, 'all the IP bands' must quantitatively be overlapped on the OP bands. ${ }^{24,31}$ As a matter of fact, however, the pMAIRS spectra have a large dichroic ratio as found for the $v(C=C)$ band (Figure 1b), meaning that the polymer-chain (long-axis) is highly ordered, since this band reflects the orientation of the long axis of the polymer-chain. In this way, pMAIRS can also be used for discriminating an isotropic structure from an oriented sample having the magic angle of $54.7^{\circ}$, which is another advantage as compared to other analytical techniques such as NEXAFS.

Of note is that the Chl-SC-8000 film exhibits an apparently better face-on orientation $\left(\phi_{\mathrm{C}-\mathrm{H}} \approx 29^{\circ}\right)$ than the conventionally known Chl-SC-4000 (33 $)$ and Chl-SC-2000 $\left(37^{\circ}\right)$ films, which is the first report to our knowledge.

In addition, the "variance" of the three points becomes smaller systematically for a higher spin speed, i.e., the variance at $8000 \mathrm{rpm}$ is smaller than those at 1000 and 
$2000 \mathrm{rpm}$ (Figure 2). This implies that the high spin-speed is suitable for a good reproducibility of the film preparation, and a homogeneous film structure.

Relationship between molecular orientation and crystallinity: Since the evaporation time should directly be correlated with the crystallinity of the film, the crystallinity of the P3HT film is discussed in detail. For this purpose, the $\gamma(\mathrm{C}-\mathrm{H})$ band of an aromatic ring is useful, since the band position responds to the molecular packing. ${ }^{24,33-36}$ For example, the band of a P3HT 'crystal' appears at $816 \mathrm{~cm}^{-1}$, while the band at $832 \mathrm{~cm}^{-1}$ is attributed to the liquid, and the two band positions are thus the extreme limits of this band region (see the upper axis of Figure 3). ${ }^{34}$ In fact, regiorandom (RRa-) P3HT known as an amorphous polymer exhibits the band at a high-wavenumber position of $827 \mathrm{~cm}^{-1},{ }^{37}$ which is relatively close to the limit of the liquid as compared to that of the crystal. The bulk solid of regioregular (RR-) P3HT used in this study, on the other hand, gives the lower-wavenumber shift by $6 \mathrm{~cm}^{-1}\left(821 \mathrm{~cm}^{-1}\right.$; spectrum not shown), which suggests that RR-P3HT has a higher crystallinity than RRa-P3HT. On this measure, the band position in the 'IP' spectrum of the P3HT thin film is discussed as follows. 


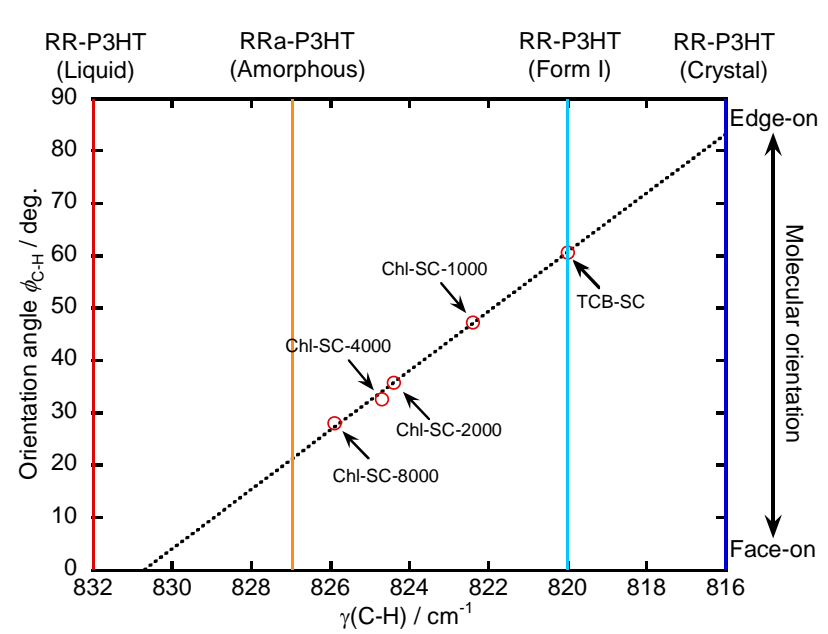

Figure 3 The orientation angle, $\phi_{\mathrm{C}-\mathrm{H}}$, as a function of the "apparent" wavenumber position of the $\gamma(\mathrm{C}-\mathrm{H})$ band in the IP spectrum. The orientation angle is calculated by using apparent band intensities without band separation.

As found in Figure 1e, the Chl-SC-8000 film has the $\gamma(\mathrm{C}-\mathrm{H})$ band at a high-wavenumber position $\left(826 \mathrm{~cm}^{-1}\right)$ that is close to the position of RRa-P3HT (827 $\mathrm{cm}^{-1}$ ), which implies that the film is nearly amorphous. The IP band of the TCB-SC film, on the other hand, exhibits a band shift to a lower position $\left(820 \mathrm{~cm}^{-1}\right.$, Figure 1a) than that of the Chl-SC film $\left(826-822 \mathrm{~cm}^{-1}\right)$ and the bulk solid $\left(821 \mathrm{~cm}^{-1}\right)$. This indicates that the TCB-SC film has a higher crystallinity than that of the Chl-SC film, and even higher than the bulk solid to our surprise. According to Yuan et al., ${ }^{38}$ the band position of 820 $\mathrm{cm}^{-1}$ corresponds to the form I structure that generally appears in the spin-coated film. In fact, the orientation angle of the TCB-SC film (61; Figure 3) agrees with the value $\left(64 \pm 5^{\circ}\right)^{39}$ expected from the form-I structure. This apparent correlation implies that a high crystallinity has a strong correlation with (or it is an origin of) the edge-on 
orientation of $\mathrm{P} 3 \mathrm{HT}$.

In fact, Figure 3 also shows, on the contrary, that the face-on orientation is generated when the crystallinity is low: the Chl-SC-8000 film has the best face-on orientation, and at the same time it has the poorest crystallinity judging from the position of the $\gamma(\mathrm{C}-\mathrm{H})$ band. This trend is confirmed by the UV-visible spectrum (Figure 4) indicating that the Chl-SC-8000 film has no shoulder band at $610 \mathrm{~nm}$ that is a useful marker band of a crystallized component. ${ }^{8,29,40-42}$ This is consistent with a fact that the band is generated by the intermolecular exciton. ${ }^{43}$ In addition, the spectrum does not show any vibronic structure when comparing to that of the TCB-SC film, which means that the polymer chain should have a disordered conformation in the thin film. ${ }^{29,44}$ According to our previous study, ${ }^{8}$ in fact, the polymer-chain is nearly disordered in a face-on oriented thin film of P3ATs involving P3HT; while the edge-on one has a highly ordered polymer-chain. The face-on orientation, in this manner, proved to be generated mainly in the amorphous region. 


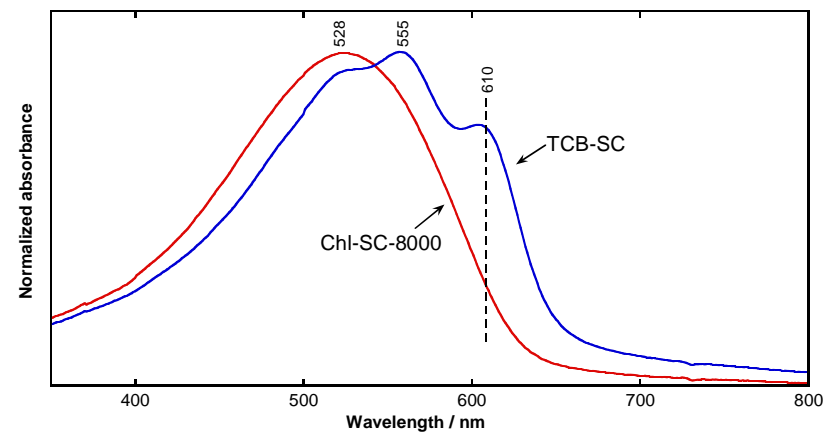

Figure 4 UV-visible spectra of the Chl-SC-8000 and the TCB-SC films prepared on $\mathrm{CaF}_{2}$ substrate.

To confirm this expectation, the band positions in the IP spectra are correlated to the orientation angles, $\phi_{\mathrm{C}-\mathrm{H}}$, for all the samples as found in Figure 3, which shows a simple linear correlation: $\phi_{\mathrm{C}-\mathrm{H}}$ decreases with increasing the wavenumber of $\gamma(\mathrm{C}-\mathrm{H})$. As discussed in the previous section, a smaller orientation angle of $\phi_{\mathrm{C}-\mathrm{H}}$ corresponds to an increase of the face-on portion. Since the wavenumber has a negative correlation with the crystallinity, ${ }^{27}$ this linear relation means that the face-on component in the film simply increases as the crystallinity decreases. In short, the face-on orientation is mainly generated in the amorphous region; whereas the edge-on orientation is induced by the crystallization of P3HT in the thin film. In other words, the face-on oriented thin film is composed of the face-on amorphous aggregates rather than the crystallites. In this manner, the $\gamma(\mathrm{C}-\mathrm{H})$ band appeared in an IP spectrum is found a very useful index to discuss the crystallinity of P3HT correlated with the orientation in a thin film.

On the other hand, the apparent position of the $\gamma(\mathrm{C}-\mathrm{H})$ band in the 'OP' 
spectrum stays at about $826 \mathrm{~cm}^{-1}$ irrespective of the evaporation time as shown in Figure 1. Since no Berreman effect ${ }^{45,46}$ is expected in this region, the difference of the band positions in the IP and OP spectra should simply be attributed to a difference of crystallinity. A significant shift between the IP and OP bands $\left(826\right.$ and $820 \mathrm{~cm}^{-1}$, respectively) is found for the TCB-SC film (Figure 1a) that is "categorized" into the edge-on orientation. The film, however, involves a portion of the face-on orientation as well as the edge-on one. On referring a fact that the form-I of P3HT yields the band at $820 \mathrm{~cm}^{-1}$, the $\gamma(\mathrm{C}-\mathrm{H})$ band at $826 \mathrm{~cm}^{-1}$ should be an "apparent" position as a result of superposition of the two bands of the amorphous and the form-I components at 827 and $820 \mathrm{~cm}^{-1}$, respectively. As a result, the TCB-SC film should comprise both face-on and edge-on portions, and the edge-on portion would not give almost $\gamma(\mathrm{C}-\mathrm{H})$ band for the OP spectrum; instead the face-on portion mainly contributes to the OP one. In this manner, the reason why the $\gamma(\mathrm{C}-\mathrm{H})$ band appears at a fixed position of $826 \mathrm{~cm}^{-1}$ in the OP spectrum has been understood.

In this sense, the Chl-SC-8000 film (Figure 1e) can be concluded to mostly consist of the face-on component only, since both IP and OP spectra have a common position of the $\gamma(\mathrm{C}-\mathrm{H})$ band $\left(826 \mathrm{~cm}^{-1}\right)$.

Effect of thermal treatment of solution on the film structure: The fact that the 
face-on orientation of $\mathrm{P} 3 \mathrm{HT}$ is generated in the amorphous region reminds us of another case of P3BT having a shorter side chain of the butyl group. Since P3BT has a relatively weak zippering effect due to the short alkyl chain, ${ }^{7}$ a P3BT film would be far from the crystal, which should prefer the face-on orientation. As a matter of fact, however, the molecular aggregation of P3BT in a 'film' is fairly strong, as found in the poor solubility in Chl due to the too short alkyl chain to have a good affinity with Chl. The poor solubility implies that the molecular 'aggregation' of P3BT is strong even in the Chl 'solution.' In fact, P3BT is known to take the edge-on orientation in a thin film even when employing a high-spin speed, ${ }^{8}$ which should be attributed to the high crystallinity of the film due to the self-aggregation property of P3BT in the solution, which is readily confirmed by measuring UV-vis spectra as shown in Figure 5.

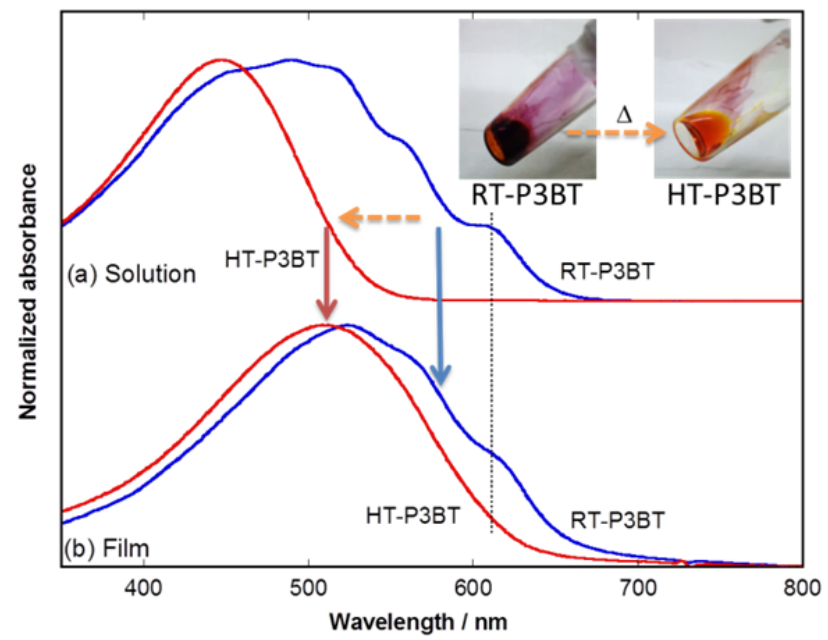

Figure 5 UV-visible spectra of RT-P3BT and HT-P3BT (a) in a Chl solution, and (b) in a thin film deposited on $\mathrm{CaF}_{2}$ substrate. Insets show pictures of the Chl solution. 
The two blue spectra in Figure 5 apparently indicate that the crystallite-marker band at $610 \mathrm{~nm}$ is clearly observed for both solution (Figure 5a) and film (Figure 5b). The edge-on orientation is thus attributed to the molecular aggregation correlated with the poor solubility at ambient temperature. If the molecular aggregates could readily be dissolved in a solvent, the face-on film would be obtained as done for P3HT. Fortunately, the P3BT aggregates in the solution are readily disaggregated only by heating the Chl solution (see inset in Figure 5), which is confirmed by the UV-vis spectra (red spectrum in Figure 5a) that the crystallite-marker band at around $610 \mathrm{~nm}$ is lost. To reveal the effect of the thermal treatment of a solution on the molecular orientation, in this section, the P3BT film prepared by using the warm solution (denoted as high-temperature (HT-) P3BT) is compared to that prepared from the solution without the thermal treatment (room temperature (RT-) P3BT).

Figure $5 \mathrm{~b}$ presents UV-vis spectra of the RT- and HT-P3BT films. The spectra show, as expected, that the HT-P3BT film has only an extremely weak intermolecular interaction as found from the fact that the shoulder band at $610 \mathrm{~nm}$ does not appear. This result is supported by the XRD patterns: the IP diffraction pattern of the RT-P3BT film clearly indicates the 100 peak at $2 \theta=7.2^{\circ}$ due to the form-I structure ${ }^{47}$ (Figure 6a); whereas the HT-P3BT film has no diffraction peaks (Figure 6b). The HT-P3BT film 
is thus found to be amorphous. In this situation, formation of the face-on oriented thin film is expected, which is readily confirmed from the pMAIRS spectra as follows.

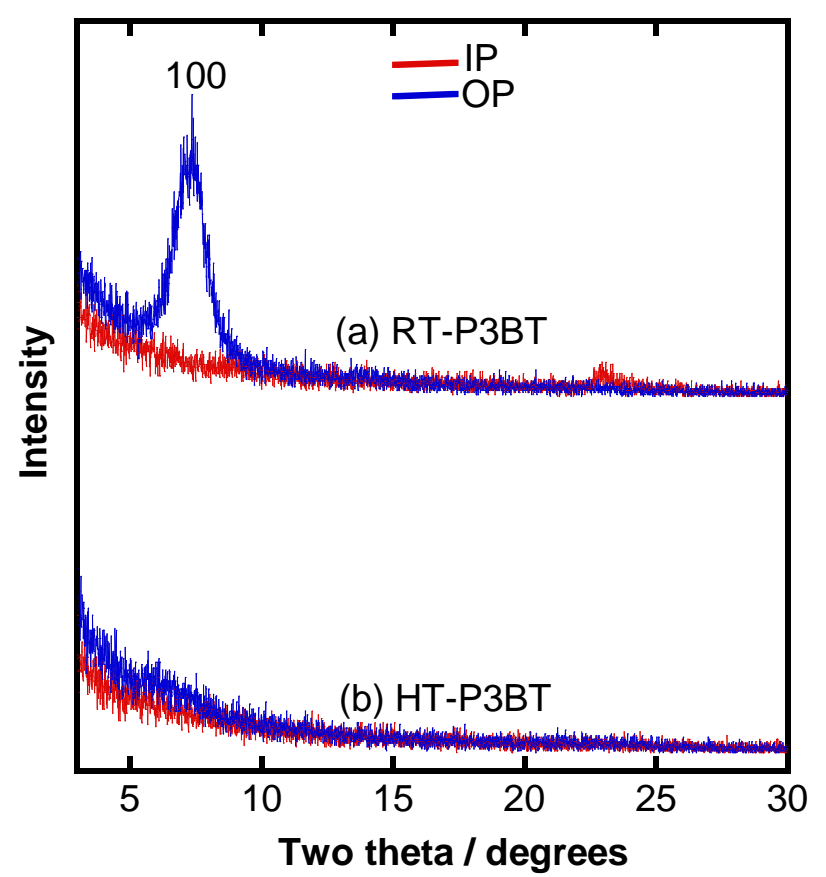

Figure 6 GIXD patterns of (a) RT- and (b) HT-P3BT thin films. The red and blue lines are measured with IP and OP geometries, respectively.

The pMAIRS spectra (Figure 7) show an obvious difference of orientation between the two films: the RT- and HT-P3BT films are categorized into the edge-on and face-on films, respectively, judging from the $\gamma(\mathrm{C}-\mathrm{H})$ band. Note that the band position of RT-P3BT $\left(825 \mathrm{~cm}^{-1}\right.$; Figure 7a) corresponds to the glassy crystal; ${ }^{33}$ while the high-wavenumber position of HT-P3BT $\left(830 \mathrm{~cm}^{-1}\right.$; Figure $\left.7 \mathrm{~b}\right)$ are nearly identical to the value $\left(832 \mathrm{~cm}^{-1}\right)$ of the amorphous state, ${ }^{48}$ which agrees with the XRD results. 


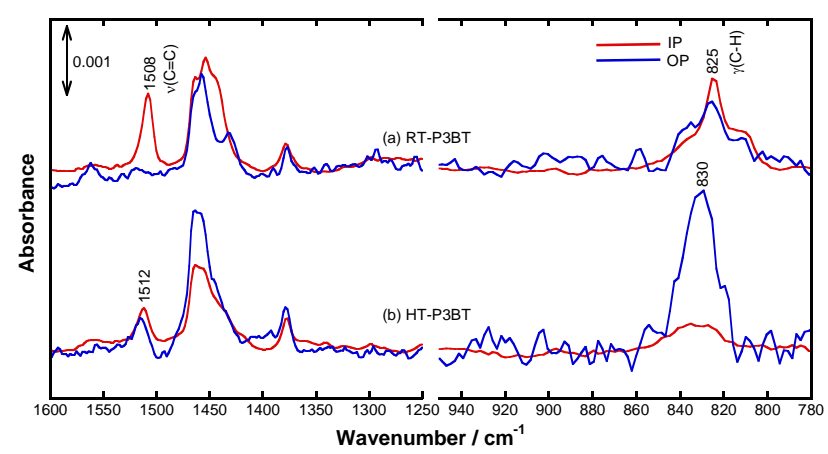

Figure 7 pMAIRS spectra of (a) RT-P3BT and (b) HT-P3BT thin films. The red and blue lines correspond to IP and OP spectra, respectively.

In addition, the dichroic ratio of the $v(\mathrm{C}=\mathrm{C})$ band between IP and OP spectra is also interesting: the RT-P3BT has a significantly large dichroic ratio; while the HT-P3BT film has a ratio of unity. Since this band responds to the orientation of the long-axis (i.e., the polymer main-chain), ${ }^{8}$ the long-axis of HT-P3BT is revealed to be disordered, although the $\gamma(\mathrm{C}-\mathrm{H})$ band is highly oriented: only the short-axis of polythiophene chain is highly kept parallel to the substrate. ${ }^{8}$ This unique schematic is a characteristic of the face-on orientation in an amorphous film. Therefore, as expected, the face-on orientation of P3BT can readily be obtained by a warmed solution, in which the molecules are disaggregated.

Of another note is that the pMAIRS-IP spectrum (Figure 7) gives the band shift of the $v(C=C)$ band, whose band position is sensitive to the conjugation length of a thiophene unit: the wavenumber has a negative correlation with the conjugation 
length. ${ }^{49}$ As can be seen in Figure 7, the RT- and HT-P3BT films have the different band positions of 1508 and $1512 \mathrm{~cm}^{-1}$, respectively, which should correspond to the shorter conjugation length of the HT-P3BT film than the RT-P3BT film. The relative short conjugation length is consistent with the low crystallinity in the HT-P3BT film.

SUMMARY: The controlling mechanism of the molecular orientation of P3AT in a thin film has been revealed by using the pMAIRS technique. The pMAIRS spectra give chemical information of both molecular orientation and crystallinity from the intensity and position of the $\gamma(\mathrm{C}-\mathrm{H})$ band of a thiophene ring, respectively. As a result, the orientation angle obtained from the pMAIRS intensity ratio is found to have a linear correlation with the band position. The linear correlation has readily revealed that the edge-on orientation is induced by crystallization of P3AT; whereas the face-on orientation is mainly generated from the amorphous region. This comprehensive understanding is true of P3BT. By using a warm solution, P3BT is readily made to take the face-on stance in an amorphous thin film. As a conclusion, the face-on orientation is not driven by forming a lamellar structure, and it is rather induced by an amorphous aggregate. 


\section{AUTHOR INFORMATION}

Corresponding Author: *E-mail: htakeshi@scl.kyoto-u.ac.jp

Notes: The authors declare no competing financial interest.

\section{ACKNOWLEDGMENTS}

This work was financially supported by a Grant-in-Aid for Scientific Research (A) (No. 15H02185 (TH)), Grant-in-Aid for Young Scientists (B) (No. 17K14502 (TS)) and Grant-in-Aid for JSPS fellows (No. 16J03487 (NS)) from the Japan Society for the Promotion of Science (JSPS), to which the authors' thanks are due.

\section{REFERENCES:}

1. Babel, A.; Jenekhe, S. Alkyl Chain Length Dependence of the Field-Effect Carrier Mobility in Regioregular Poly(3-alkylthiophene)s. Synth. Met. 2005, 148, 169-173.

2. Nguyen, L. H.; Hoppe, H.; Erb, T.; Günes, S.; Gobsch, G.; Sariciftci, N. S. Effects of Annealing on the Nanomorphology and Performance of Poly(alkylthiophene):Fullerene Bulk-Heterojunction Solar Cells. Adv. Funct. Mater. 2007, 17, 1071-1078. 
3. Huang, W. Y.; Lee, C. C.; Wang, S. G.; Han, Y. K.; Chang, M. Y. Side Chain Effects of Poly(3-alkylthiophene) on the Morphology and Performance of Polymer Solar Cells. J. Electrochem. Soc. 2010,157, B1336-B1342.

4. Friedel, B.; McNeill, C. R.; Greenham, N. C. Influence of Alkyl Side-Chain Length on the Performance of Poly(3-alkylthiophene)/Polyfluorene All-Polymer Solar Cells. Chem. Mater. 2010, 22, 3389-3398.

5. Zaumseil, J; Sirringhaus, H. Electron and Ambipolar Transport in Organic Field-Effect Transistors. Chem. Rev. 2007, 107, 1296-1323.

6. Günes, S.; Neugebauer, H.; Sariciftci, N. S. Conjugated Polymer-Based Organic Solar Cells. Chem. Rev. 2007, 107, 1324-1338.

7. Tashiro, K.; Minagawa, Y.; Kobayashi, M.; Morita, S.; Kawai, T.; Yoshino, K. Structure and Thermochromic Solid-State Phase Transition of Poly (3-alkylthiophene). Stnth. Met. 1993, 55-57, 321-328.

8. Shioya, N.; Shimoaka, T.; Eda, K.; Hasegawa, T. A New Schematic for Poly(3-alkylthiophene) in an Amorphous Film Studied Using a Novel Structural Index in Infrared Spectroscopy. Phys. Chem. Chem. Phys. 2015, 17, 13472-13479.

9. Brinkmann, M.; Wittmann, J.-C. Orientation of Regioregular Poly(3-hexylthiophene) by Directional Solidification: A Simple Method to Reveal 
the Semicrystalline Structure of a Conjugated Polymer. Adv. Mater. 2006, 18, 860863.

10. Duong, D. T.; Toney, M. F.; Salleo, A. Role of Confinement and Aggregation in Charge Transport in Semicrystalline Polythiophene Thin Films. Phys. Rev. B 2012, $86,205205$.

11. Hugger, S.; Thomann, R.; Heinzel, T.; Thurn-Albrecht, T. Semicrystalline Morphology in Thin Films of Poly(3-hexylthiophene). Colloid. Polym. Sci. 2004, 282, 932-938.

12. Sirringhaus, H.; Brown, P.; Friend, R.; Nielsen, M. M.; Bechgaard, K.; Langeveld-Voss, B. M. W.; Spiering, A. J. H.; Janssen, R. A. J.; Meijer, E. W.; Herwig, P.; Leeuw, D. M. Two-Dimensional Charge Transport in Self-Organized, High-Mobility Conjugated Polymers. Nature 1999, 401, 685-688.

13. Yang, H.; LeFevre, S. W.; Ryu, C. Y.; Bao, Z. Solubility-Driven Thin Film Structures of Regioregular Poly(3-hexyl thiophene) Using Volatile Solvents. Appl. Phys. Lett. 2007, 90, 172116.

14. Hasegawa, T. Advanced Multiple-Angle Incidence Resolution Spectrometry for Thin-Layer Analysis on a Low-Refractive-Index Substrate. Anal. Chem. 2007, 79, $4385-4389$. 
15. Hasegawa, T. A Novel Measurement Technique of Pure Out-of-Plane Vibrational Modes in Thin Films on a Nonmetallic Material with No Polarizer. J. Phys. Chem. B 2002, 106, 4112-4115.

16. Shioya, N.; Norimoto, S.; Izumi, N.; Hada, M.; Shimoaka, T.; Hasegawa, T. Optimal Experimental Condition of IR pMAIRS Calibrated by Using an Optically Isotropic Thin Film Exhibiting the Berreman Effect. Appl. Spectrosc. 2017, 71, 901-910.

17. Drapcho, D.; Hasegawa, T. Applications of Infrared Multiple Angle Incidence Resolution Spectrometry. Spectroscopy 2015, 30, 31-38.

18. Kakuda, H.; Okada, T.; Hasegawa, T. Anisotropic Molecular Structure in Dip-Coated Films of Linear Poly(ethylene imine) Studied by Infrared Multiple-Angle Incidence Resolution Spectrometry. J. Phys. Chem. B 2008, 112, $12940-12945$.

19. Nagao, Y. Highly Oriented Sulfonic Acid Groups in a Nafion Thin Film on Si Substrate. J. Phys. Chem. C 2013, 117, 3294-3297.

20. Kline, R. J.; McGehee, M. D.; Toney, M. F. Highly Oriented Crystals at the Buried Interface in Polythiophene Thin-Film Transistors. Nat. Mater. 2006, 5, 222-228. 
21. Kitchen, B.; Awartani, O.; Kline, R. J.; McAfee, T.; Ade, H.; O’Connor, B. T. Tuning Open-Circuit Voltage in Organic Solar Cells with Molecular Orientation. ACS Appl. Mater. Interfaces 2015, 7, 13208-13216.

22. Shao, S.; Liu, J.; Zhang, J.; Zhang, B.; Xie, Z.; Geng, Y.; Wang, L. Interface-Induced Crystalline Ordering and Favorable Morphology for Efficient Annealing-Free Poly(3-hexylthiophene): Fullerene Derivative Solar Cells. ACS Appl. Mater. Interfaces 2012, 4, 5704-5710.

23. Vohra, V.; Arrighetti, G.; Barba, L.; Higashimine, K.; Porzio, W.; Murata, H. Enhanced Vertical Concentration Gradient in Rubbed P3HT:PCBM Graded Bilayer Solar Cells. J. Phys. Chem. Lett. 2012, 3, 1820-1823.

24. Hada, M.; Shioya, N.; Shimoaka, T.; Eda, K.; Hada, M.; Hasegawa, T. Comprehensive Understanding of Structure-Controlling Factors of a Zinc Tetraphenylporphyrin Thin Film Using pMAIRS and GIXD Techniques. Chem. Eur. J. 2016, 22, 16539-16546.

25. Chabinyc, M. L. X-ray Scattering from Films of Semiconducting Polymers. Polym. Rev. 2008, 48, 463-492.

26. Chang, J.-F.; Sun, B.; Breiby, D. W.; Nielsen, M. M.; Sölling, T. I.; Giles, M.; McCulloch, I.; Sirringhaus, H. Enhanced Mobility of Poly(3-hexylthiophene) 
Transistors by Spin-Coating from High-Boiling-Point Solvents. Chemistry of Materials 2004, 16, 4772-4776.

27. Shioya, N.; Shimoaka, T.; Hasegawa, T. Analysis of Molecular Orientation and Conformation of Poly(3-hexylthiophene) Thin Films on Silicon by Infrared p-Polarized Multiple-angle Incidence Resolution Spectrometry. Chem. Lett. 2014, 43, 1198-1200.

28. DeLongchamp, D. M.; Vogel, B. M.; Jung, Y.; Gurau, M. C.; Richter, C. A.; Kirillov, O. A.; Obrzut, J.; Fischer, D. A.; Sambasivan, S.; Richter, L. J.; Lin, E. K. Variations in Semiconducting Polymer Microstructure and Hole Mobility with Spin-Coating Speed. Chem. Mater. 2005, 17, 5610-5612.

29. Hamidi-Sakr, A.; Biniek, L.; Fall, S.; Brinkmann, M. Precise Control of Lamellar Thickness in Highly Oriented Regioregular Poly(3-Hexylthiophene) Thin Films Prepared by High-Temperature Rubbing: Correlations with Optical Properties and Charge Transport. Adv. Funct. Mater. 2016, 26, 408-420.

30. Nagamatsu, S.; Takashima, W.; Kaneto, K.; Yoshida, Y.; Tanigaki, N.; Yase, K.; Omote, K. Backbone Arrangement in "Friction-Transferred" Regioregular Poly(3-alkylthiophene)s. Macromolecules 2003, 36, 5252-5257. 
31. Shioya, N.; Shimoaka, T.; Murdey, R.; Hasegawa, T. Accurate Molecular Orientation Analysis Using Infrared P-Polarized Multiple-Angle Incidence Resolution Spectrometry (pMAIRS) Considering the Refractive Index of the Thin Film Sample. Appl. Spectrosc. 2017, DOI: 10.1177/0003702816676492.

32. Clark, J.; Chang, J.-F.; Spano, F. C.; Friend, R. H.; Silva, C. Determining Exciton Bandwidth and Film Microstructure in Polythiophene Films Using Linear Absorption Spectroscopy. Appl. Phys. Lett. 2009, 94, 163306.

33. Yazawa, K.; Inoue, Y.; Yamamoto, T.; Asakawa, N. Twist Glass Transition in Regioregulated poly(3-alkylthiophene). Phys. Rev. B 2006, 74, 094204.

34. Yazawa, K.; Inoue, Y.; Yamamoto, T.; Asakawa, N. Dynamic Structure of Regioregulated Poly(alkylthiophene)s. J. Phys. Chem. B 2008, 112, 11580-11585.

35. Westermeier, C.; Cernescu, A.; Amarie, S.; Liewald, C.; Keilmann, F.; Nickel, B. Sub-Micron Phase Coexistence in Small-Molecule Organic Thin Films Revealed by Infrared Nano-Imaging. Nat. Commun. 2014, 5, 4101.

36. Sharp, J.; Abkowitz, M. Dimeric Structure of a Copper Phthalocyanine Polymorph. J. Phys. Chem. 1973, 77, 477-481. 
37. Chen, T.-A.; Wu, X.; Rieke, R. D. Regiocontrolled Synthesis of Poly(3-alkylthiophenes) Mediated by Rieke Zinc: Their Characterization and Solid-State Properties. J. Am. Chem. Soc. 1995, 117, 233-244.

38. Yuan, Y.; Zhang, J.; Sun, J.; Hu, J.; Zhang, T.; Duan, Y. Polymorphism and Structural Transition around $54{ }^{\circ} \mathrm{C}$ in Regioregular Poly(3-hexylthiophene) with High Crystallinity As Revealed by Infrared Spectroscopy. Macromolecules 2011, 44, 9341-9350.

39. Kayunkid, N.; Uttiya, S.; Brinkmann, M. Structural Model of Regioregular Poly(3-hexylthiophene) Obtained by Electron Diffraction Analysis. Macromolecules 2010, 43, 4961-4967.

40. Trznadel, M.; Pron, A.; Zagorska, M.; Chrzaszcz, R.; Pielichowski, J. Effect of Molecular Weight on Spectroscopic and Spectroelectrochemical Properties of Regioregular Poly(3-hexylthiophene). Macromolecules 1998, 31, 5051-5058.

41. Zen, A; Pflaum, J; Hirschmann, S.; Zhuang, W.; Jaiser, F.; Asawapiron, U.; Rabe, J. P.; Scherf, U.; Neher, D. Effect of Molecular Weight and Annealing of Poly(3-hexylthiophene)s on the Performance of Organic Field-Effect Transistors. Adv. Funct. 2004, 14, 757-764. 
42. Kanai, K.; Miyazaki, T.; Suzuki, H.; Inaba, M.; Ouchi, Y.; Seki, K. Effect of Annealing on the Electronic Structure of Poly(3-hexylthiophene) Thin Film. Phys. Chem. Chem. Phys. 2009, 12, 273-282.

43. Brown, P. J.; Thomas, D. S.; Köhler, A.; Wilson, J. S.; Kim, J.-S.; Ramsdale, C. M.; Sirringhaus, H.; Friend, R. H. Effect of Interchain Interactions on the Absorption and Emission of Poly(3-hexylthiophene). Phys. Rev. B 2003, 67, 064203.

44. Spano, F. C. Modeling Disorder in Polymer Aggregates: the Optical Spectroscopy of Regioregular Poly(3-hexylthiophene) Thin Films. J. Chem. Phys. 2005, 122, 234701

45. Berreman, D. W. Infrared Absorption at Longitudinal Optic Frequency in Cubic Crystal Films. Phys. Rev. 1963, 130, 2193.

46. Yamamoto, K; Masui, A TO-LO Splitting in Infrared Spectra of Thin Films. Appl. Spectrosc. 1996, 50, 759-763.

47. Buono, A.; Son, N.; Raos, G.; Gila, L.; Cominetti, A.; Catellani, M.; Meille, S. Form II Poly(3-butylthiophene): Crystal Structure and Preferred Orientation in Spherulitic Thin Films. Macromolecules 2010, 43, 6772-6781. 
48. Yuan, Y.; Shu, J.; Kolman, K.; Kiersnowski, A.; Bubeck, C.; Zhang, J.; Hansen, M. Multiple Chain Packing and Phase Composition in Regioregular Poly(3-butylthiophene) Films. Macromolecules 2016, 49, 9493-9506.

49. Hernández, V.; Casado, J.; Ramírez, F. J.; Zotti, G.; Hotta, S.; Navarrete, J. T. L. Efficient $\pi$ Electrons Delocalization in $\alpha, \alpha^{\prime}$-dimethyl End-Capped Oligothiophenes: A Vibrational Spectroscopic Study. J. Chem. Phys. 1996, 104, 9271-9282. 